\title{
Production of reactive oxygen species by PuRBOHF is critical for stone cell development in pear fruit
}

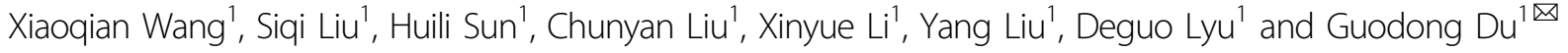

\begin{abstract}
The production of reactive oxygen species (ROS) by NADPH oxidase, which is also referred to as respiratory burst oxidase homolog $(\mathrm{RBOH})$, affects several processes in plants. However, the role of RBOHs in cell wall lignification is not well understood. In this study, we show that PuRBOHF, an $\mathrm{RBOH}$ isoform, plays an important role in secondary wall formation in pear stone cells. ROS were closely associated with lignin deposition and stone cell formation according to microscopy data. In addition, according to the results of an in situ hybridization analysis, the stage-specific expression of PURBOHF was higher in stone cells than in cells of other flesh tissues. Inhibitors of RBOH activity suppressed ROS accumulation and stone cell lignification in pear fruit. Moreover, transient overexpression of PURBOHF caused significant changes in the amount of ROS and lignin that accumulated in pear fruit and flesh calli. We further showed that PUMYB169 regulates PURBOHF expression, while PURBOHF-derived ROS induces the transcription of PuPOD2 and PuLAC2. The findings of this study indicate that PURBOHF-mediated ROS production, which is regulated by a ligninrelated transcriptional network, is essential for monolignol polymerization and stone cell formation in pear fruit.
\end{abstract}

\section{Introduction}

Pear (a member of the Pyrus genus), which belongs to the Rosaceae family, is an important fruit tree species and is widely cultivated throughout temperate regions worldwide. At least 22 Pyrus species have been identified worldwide $^{1}$, among which five species, $P$. ussuriensis, P. pyrifolia, P. bretschneideri, P. communis, and P. sinkiangensis, are the most cultivated ${ }^{2}$. Unlike the fruit of other Pyrus species, P. ussuriensis fruit has a relatively high stone cell content, which is a crucial factor affecting fruit quality ${ }^{3}$. The presence of stone cells contributes to the rough flesh texture of some pear fruit, thereby reducing their economic value ${ }^{4}$. Therefore, it is essential to reduce the content of stone cells to improve pear quality.

Pear stone cells are sclerenchyma cells formed by the secondary deposition of lignin within the primary walls of parenchyma cells ${ }^{5}$. The formation of stone cells is strongly associated with the synthesis, transfer, and deposition of

Correspondence: Guodong Du (guodongdu@syau.edu.cn)

${ }^{1}$ College of Horticulture, Shenyang Agricultural University, Shenyang 110866,

P. R. China

These authors contributed equally: Xiaoqian Wang, Siqi Liu, Huili Sun lignin in pear flesh ${ }^{6}$. The lignin polymers in pear mainly comprise two monolignols, coniferyl, and sinapyl alcohols $^{7}$. As the main building blocks of lignin polymers, monolignols are synthesized via phenylpropanoid- and monolignol-specific pathways ${ }^{8}$. Several enzymes involved in this pathway have been identified ${ }^{9-11}$. Xue et al. ${ }^{4}$ reported that PbrMYB169, a MYB transcription factor, positively regulates monolignol biosynthesis in pear fruit. After monolignols are formed, they are translocated to the cell wall where they are polymerized by peroxidase and laccase $^{12}$. In addition to the lignin monomers, peroxidases, and laccases required to form monolignol radicals, additional substrates such as hydrogen peroxide $\left(\mathrm{H}_{2} \mathrm{O}_{2}\right)$ and molecular oxygen $\left(\mathrm{O}_{2}\right)$ are required ${ }^{13}$. However, the source of reactive oxygen species (ROS) required for the peroxidase and laccase activity remains unknown.

Although early research focused on the toxicity of ROS, interest has shifted toward their role as signaling molecules in a wide range of physiological processes, such as seed germination, root growth, stomatal opening, pollen tube elongation, and aerenchyma formation ${ }^{14-20}$. ROS signaling also plays an important role in plant lignification.

\section{(c) The Author(s) 2021}

(c) (i) Open Access This article is licensed under a Creative Commons Attribution 4.0 International License, which permits use, sharing, adaptation, distribution and reproduction cc) in any medium or format, as long as you give appropriate credit to the original author(s) and the source, provide a link to the Creative Commons license, and indicate if changes were made. The images or other third party material in this article are included in the article's Creative Commons license, unless indicated otherwise in a credit line to the material. If material is not included in the article's Creative Commons license and your intended use is not permitted by statutory regulation or exceeds the permitted use, you will need to obtain permission directly from the copyright holder. To view a copy of this license, visit http://creativecommons.org/licenses/by/4.0/. 
In Arabidopsis, the scaffolding of NADPH oxidases to downstream targets of ROS produced might enable localized lignin deposition in the endodermis ${ }^{21}$. Furthermore, exogenous $\mathrm{H}_{2} \mathrm{O}_{2}$ was shown to increase the lignin content in rice roots, whereas diphenyleneiodonium chloride (DPI) inhibited this phenomenon ${ }^{22}$. Heng et al. ${ }^{23}$ found that $\mathrm{H}_{2} \mathrm{O}_{2}$ from polyamine metabolism affects lignification in pear exocarp tissue. However, a molecularly defined signaling pathway through which ROS production in pear fruit in induced during lignification has not been identified.

NADPH oxidases, which are also referred to as respiratory burst oxidase homologs (RBOHs) and are localized in the cell plasma membrane, are the most extensively studied sources of extracellular ROS in plants $^{24}$. In addition to being pivotal for defense against biotic and abiotic stresses, various RBOHs control a large number of developmental processes in response to both internal and external stimuli ${ }^{25}$. In plants, $R B O H s$ constitute a multigene family, and each homolog has a specific role. In Arabidopsis, AtRBOHB regulates seed after ripening $^{26}$, AtRBOHC regulates root hair formation ${ }^{27}$, and AtRBOHE regulates anther and pollen development ${ }^{18}$. Moreover, some RBOHs mediate ROS production during lignin production. In pear, three $\mathrm{PbRBOH}$ components are closely related to known lignificationrelated $R B O H \mathrm{~s}^{28}$. However, their functionality has yet to be validated.

Lignification is characterized by an increase in ROS production, which might act as a developmental signal in secondary wall differentiation ${ }^{17}$. Although some studies have suggested the involvement of $\mathrm{RBOH}$ in the lignification of pear stone cells, it remains unclear which $\mathrm{RBOH}$ isoforms are involved and how the ROSproducing system interacts with the lignin-related transcriptional network. This study was conducted to identify the $\mathrm{RBOH}$ genes involved in stone cell lignification and to clarify the molecular mechanisms underlying ROS signaling pathways. Our results will provide guidance for improving fruit quality through a reduction in the content of stone cells.

\section{Materials and methods \\ Plant materials}

Sixty-year-old P. ussuriensis 'Nanguo' trees in an orchard at Shenyang Agriculture University, Shenyang, China, were used as materials. Fruit samples were collected at 20,40, 55, 65, 100, and 130 days after full bloom (DAFB). Fresh fruits were used for histochemical analysis, and some fruits were stored at $-80^{\circ} \mathrm{C}$ for further analysis. Three replicates were included in this study, and each biological replicate comprised ten fruits. Fresh roots, stems, leaves, and flowers were sampled from the same plant.

\section{Analysis of stone cell and lignin contents}

The stone cell content was determined via a frozen $\mathrm{HCl}$ treatment as previously described ${ }^{29}$ and was calculated as follows: stone cell content $(\%)=$ stone cell dry weight $($ g DW)/flesh fresh weight $(\mathrm{g} F W) \times 100$. The acetyl bromide method was used to estimate the lignin content as described by Anderson et al. ${ }^{30}$. The lignin content was expressed as a percentage and calculated as follows: lignin content $(\%)=$ calculated lignin content/calculated DW $\times 100$.

\section{Lignin histochemical assays}

Hand-cut sections were prepared from fresh pear fruit tissues to confirm the presence of stone cell clusters in the fruit flesh. The sections were placed in a phloroglucinol solution for $10 \mathrm{~min}$ and then treated with $30 \% \mathrm{HCl}$ for $5 \mathrm{~min}$. The stained sections were imaged using a handheld camera. For microscopy analysis, sample preparation, including sample fixing, embedding, and sectioning, was performed as described by Tao et al. ${ }^{31}$. The sections were stained with phloroglucinol- $\mathrm{HCl}$ and examined using an optical microscope.

\section{Determination of the $\mathrm{H}_{2} \mathrm{O}_{2}$ content}

The $\mathrm{H}_{2} \mathrm{O}_{2}$ content was determined by measuring the production of $\mathrm{H}_{2} \mathrm{O}_{2}$-titanium complexes formed through the reaction of tissue $\mathrm{H}_{2} \mathrm{O}_{2}$ with titanium tetrachloride ${ }^{32}$. The $\mathrm{H}_{2} \mathrm{O}_{2}$ levels were measured at $415 \mathrm{~nm}$ and quantified using a standard curve.

\section{Histochemical assays for ROS}

The $\mathrm{H}_{2} \mathrm{O}_{2}$ level in flesh tissues was monitored using DAB (3,3-diaminobenzidine)-stained sections following the methods described by Choi et al. ${ }^{33}$. Hand-cut fruit sections were subsequently dipped in $\mathrm{DAB}-\mathrm{HCl}$ solution and incubated in the dark at room temperature. Then, the samples were boiled in $90 \%$ ethanol for $10 \mathrm{~min}$ for bleaching. The stained sections were subsequently imaged with a hand-held camera. In situ detection of $\mathrm{H}_{2} \mathrm{O}_{2}$ was performed using the fluorescent dye $2^{\prime}, 7^{\prime}$-dichlorodihydrofluorescein diacetate $\left(\mathrm{H}_{2}\right.$ DCF-DA) staining method according to the methods of Chen et al. ${ }^{34}$. The pear fruit tissue was cut into $10 \mu \mathrm{m}$ sections for histological examination using a sliding freezing microtome (CM1850, Leica, Germany) and incubated with a $10 \mu \mathrm{M}$ solution of $\mathrm{H}_{2}$ DCF-DA for $5 \mathrm{~min}$ in the dark. The accumulation of $\mathrm{H}_{2} \mathrm{O}_{2}$ was observed as green fluorescence using a confocal microscope (TCS SP8, Leica, Germany).

\section{Transmission electron microscopy}

In situ detection of subcellular ROS localization was conducted via transmission electron microscopy with cerium chloride $\left(\mathrm{CeCl}_{3}\right)$ staining. The pear flesh was cut into small $\left(1-2 \mathrm{~mm}^{3}\right)$ pieces and then incubated in 
$50 \mathrm{mM}$ 3-(N-morpholino)propanesulfonic acid (MOPS) containing $5 \mathrm{mM} \mathrm{CeCl}_{3}$ at $\mathrm{pH} 7.2$ for $1 \mathrm{~h}$. The samples were then dehydrated and fixed based on the methods of Bestwick et al. ${ }^{35}$.

\section{Chemical treatments}

To evaluate the role of NADPH oxidase as a source of ROS production during lignin biosynthesis, the flesh of Nanguo pear fruit at 40 DAFB were injected with $\mathrm{H}_{2} \mathrm{O}_{2}$ $\left(0.1 \mathrm{ml}\right.$ of $\left.500 \mu \mathrm{M} \mathrm{H} \mathrm{H}_{2} \mathrm{O}_{2}\right)$ and diphenyleneiodonium chloride (20 $\mu \mathrm{M}$ DPI; an NADPH oxidase inhibitor) using a syringe without a needle. Pear fruit injected with distilled water served as controls. After 10 days, flesh tissue from the injection sites was collected for histochemical and further analyses.

To examine the response pattern of PuRBOHF to various abiotic stresses and hormone treatments, Nanguo pear fruit at 40 DAFB were used. For chilling treatment, the fruits were incubated at $4{ }^{\circ} \mathrm{C}$ in a dark growth chamber. For the wounding treatment, scissors were used to make cuts $\sim 0.5 \mathrm{~cm}$ in length in the peel. For hormone treatment, the fruits were immersed in abscisic acid (ABA), salicylic acid (SA), and ethephon (ETH) solutions for $15 \mathrm{~min}$. Untreated fruits were used as controls. For the control, wounding, and hormone treatments, the fruits were incubated for $24 \mathrm{~h}$ in the dark in a growth chamber at $18{ }^{\circ} \mathrm{C}$ and $70 \%$ relative humidity. The flesh tissue was then collected for qRT-PCR analysis.

\section{Measurement of NADPH oxidase activity}

Plasma membranes were prepared according to the procedure described by Zhang et al $^{36}$. The activity of plasma membrane NADPH oxidase was determined based on the reduction of 2,3-bis(2-methoxy-4-nitro-5sulfophenyl)-2H-tetrazolium-5- carboxanilideinner salt (XTT) by $\mathrm{O}^{2}-$ radicals as described by Sagi and Fluhr ${ }^{37}$. The assay reaction media included $50 \mu \mathrm{l}$ of enzyme extract, $0.5 \mathrm{mM}$ XTT, $0.1 \mathrm{mM} \mathrm{NADPH}, 2.0 \mathrm{mM} \mathrm{CaCl} 2$ and $100 \mathrm{mM}$ Tris- $\mathrm{HCl}(\mathrm{pH}$ 7.5). XTT reduction was monitored at $470 \mathrm{~nm}$ in the presence or absence of 50 units of SOD.

Isolation of developing stone cells from fruit flesh tissues

Stone cells were isolated from flesh tissues at 40 DAFB according to the method of Xue et al. ${ }^{4}$. The frozen flesh samples were thawed in RNase-free water at $4{ }^{\circ} \mathrm{C}$ and then incubated on ice for $30 \mathrm{~min}$ with periodic vortexing. After centrifugation, the stone cells were resuspended in RNase-free water that included glycine, collected via slow centrifugation, and stored at $-80^{\circ} \mathrm{C}$ for RNA extraction.

\section{Expression profiles of genes}

Total RNA was extracted using the cetyltrimethylammonium bromide (CTAB)-based method.
First-strand cDNA was synthesized using a PrimeScript RT Reagent Kit (Takara, Tokyo, Japan) according to the kit instructions. qRT-PCR was performed on a 7500 Realtime PCR system (Applied Biosystems, Foster City, USA) using a SYBR Green Kit (Takara, Tokyo, Japan). PuActin served as the internal control. The primers used are listed in Table S1.

\section{Subcellular localization}

The vector construction and experimental procedures for determining the subcellular location of PuRBOHF were performed according to the methods of Cheng et $\mathrm{al}^{38}$. The PuRBOHF coding region was fused in frame to the green fluorescent protein (GFP) $\mathrm{N}$-terminus to form a CaMV35S-PuRBOHF-GFP fusion vector. The fusion constructs were transferred into Agrobacterium tumefaciens strain GV1301 via the freeze-thaw method. The leaves of Nicotiana benthamiana plants were infiltrated through their abaxial surfaces with the Agrobacterium suspension. At $72 \mathrm{~h}$ post-infiltration, the whole leaf tissues of the infiltration sites were collected, stained with DAPI, and examined using a confocal microscope (TCS SP8, Leica, Germany).

\section{RNA in situ hybridization}

Flesh tissues were collected from pear fruit at 40 DAFB, fixed in formaldehyde solution for $12 \mathrm{~h}$ at $4{ }^{\circ} \mathrm{C}$, embedded in paraffin, and cut into $7 \mu \mathrm{m}$ thick sections. The sections were subsequently hybridized, and probes were detected with NBT (nitro blue tetrazolium chloride) according to a previously described procedure ${ }^{18}$.

\section{Transient expression of pear fruit and fruit calli}

A $p$ CAMBIA1301S-PuRBOHF overexpression vector was obtained by cloning the ORF of $P U R B O H F$ into a $p C A M B I A 1301 S$ vector. To generate PuRBOHF-silenced constructs, a 250-bp fragment of $P u R B O H F$ was amplified and cloned into a $p F G C 5941$ vector. The primers used are shown in Table S2. The constructs were then transformed into Agrobacterium tumefaciens strain GV1301 using a previously described method. The transformed Agrobacterium cells were then injected into the flesh of Nanguo pear fruit at 40 DAFB using needleless syringes ${ }^{39}$. Thirty fruits were injected with each construct. The injected fruits were left on the tree for 10 days under lowlight conditions imposed by shading. Then, the fruits were randomly collected for histochemical analysis and other measurements.

Pear calli were induced from the flesh of young Nanguo fruit according to the methods of Bai et al. ${ }^{40}$. The transformation of pear calli was performed as follows: fruit calli were soaked in Agrobacterium suspensions for $10 \mathrm{~min}$. After 3 days of coculture, the calli were screened using hygromycin B on MS solid media in the dark. Then, the 
lignin content of the transformed calli was determined as previously described.

\section{GUS analysis}

A GUS reporter gene was employed to study the effects of $\mathrm{H}_{2} \mathrm{O}_{2}$ on the expression patterns of the PuPOD2 and PuLAC2 genes according to the methods of Wu et al. ${ }^{41}$. The promoters of PuPOD2 and PuLAC2 were fused to a $p B I 101-G U S$ vector to generate the proPuPOD2-GUS and proPuLAC2-GUS reporters, respectively. Tobacco leaves were used for transient GUS activity assays. Agrobacterium tumefaciens strain GV1301 cells containing each of these two reporters were infiltrated into tobacco leaves (5-week old). For $\mathrm{H}_{2} \mathrm{O}_{2}$ treatment, infiltrated tobacco leaves were sprayed with a $1 \mathrm{mM} \mathrm{H}_{2} \mathrm{O}_{2}$ solution, and those treated with distilled water were used as controls. Histochemical GUS staining was performed as described by $\mathrm{He}$ et al. ${ }^{42}$. Fluorometric determination of GUS activity was conducted as previously described ${ }^{43}$.

\section{Dual-luciferase assays}

A dual-luciferase reporter assay was used to verify the interaction between PuMYB169 and the PuRBOHF promoter $^{44}$. To generate a reporter construct, the PuRBOHF promoter region was amplified via PCR using the specific primers listed in Table S2. The PCR product was subsequently fused into a $p$ Green II 0800-LUC vector, yielding proPuRBOHF-LUC. To generate an effector construct, the full-length PuMYB169 coding sequence was inserted into a pCAMBIA1300S vector to generate a 35S-PuMYB169 fusion vector. All the constructed vectors were transformed into Agrobacterium strain GV1301. Mixtures or only reporters of Agrobacterium strains were introduced into tobacco leaves. A fluorescence assay was performed after three days using a Tanon 5200 Multi-Imaging System (Tanon, Shanghai, China). LUC/REN activity was determined using a dual-luciferase reporter assay kit (Beyotime, Jiangsu, China).

\section{Statistical analysis}

All the data were analyzed using SPSS 17.0. Student's two-tailed $t$ test $(P<0.05)$ was used to determine differences between the two groups. The figures were generated via Origin 2019b, and phylogenetic analysis was conducted using MEGA 5.0.

\section{Results}

\section{Accumulation of lignin and ROS during stone cell formation}

Microscopy analysis was performed to determine the distribution and size of stone cells in the flesh of the pear fruit. As observed via light microscopy at 65 DAFB (Fig. S1), lignified stone cells were stained deep red. Most of the stone cells developed as clusters scattered

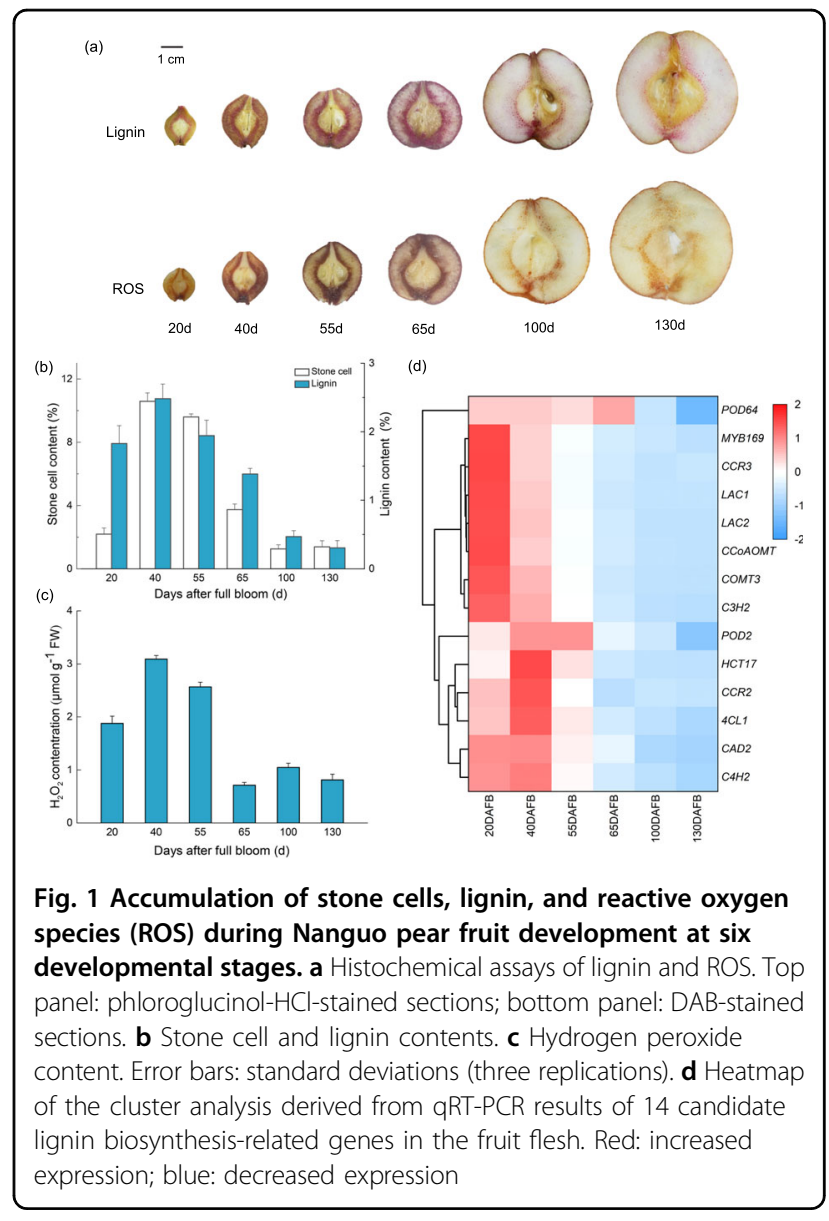

irregularly throughout the fruit flesh. Secondary thickening and primary cell wall lignification are important features of pear stone cells. In the TEM images, various cell wall layers could be clearly identified because of the high lignin concentrations in those regions. As cell wall thickening progressed, the plastids and vacuoles shrank.

To investigate the roles of ROS in the development of pear stone cells, hand-cut sections were stained with phloroglucinol- $\mathrm{HCl}$ and $\mathrm{DAB}$. The accumulation of lignin and ROS in the samples tended to be similar, with an initial increase in the staining area followed by a decrease during the course of fruit development (Fig. 1a). In addition, stone cell, lignin and $\mathrm{H}_{2} \mathrm{O}_{2}$ contents were measured during fruit development. The relative contents of stone cells and lignin on a fresh-weight (FW) basis exhibited a quadratic pattern, peaking at 40 DAFB and decreasing thereafter (Fig. 1b). Similarly, the $\mathrm{H}_{2} \mathrm{O}_{2}$ content of the fruit exhibited a trend almost the same as that of lignin; however, the lowest $\mathrm{H}_{2} \mathrm{O}_{2}$ content was observed at 65 DAFB (Fig. 1c).

The expression levels of structural genes involved in the lignin biosynthesis pathway (C4H2, 4CL1, HCT17, C3H2, CCOAOMT, COMT3, CCR2, CCR3, CAD2, LAC1, LAC2, 


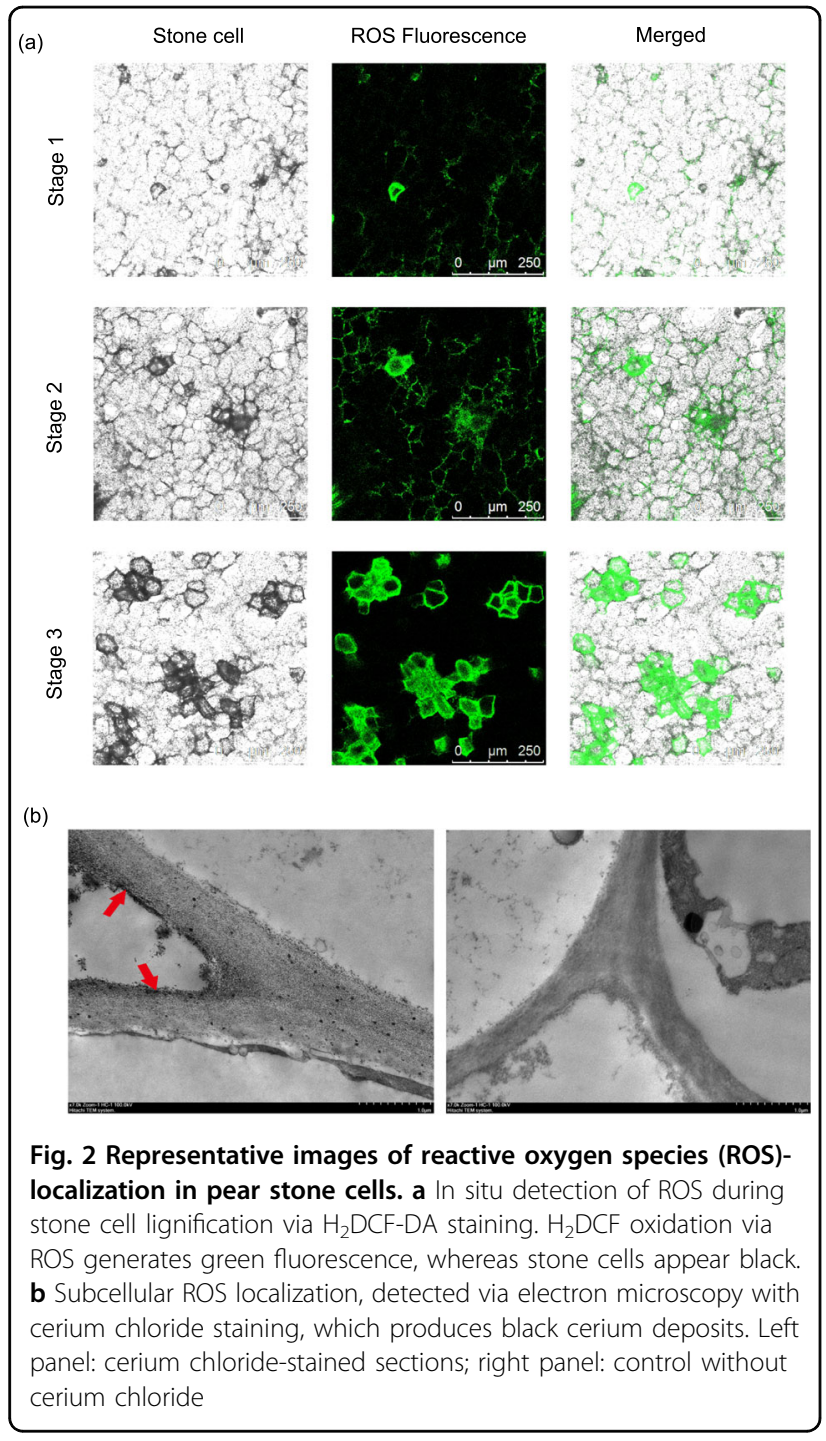

$P O D 2$, and POD64) and related transcription factors (MYB169) were also analyzed in the flesh of Nanguo pear fruit during fruit development (Fig. 1d). Except for CCR2, these genes were highly expressed in the early stages and weakly expressed in later stages, which is consistent with the changes in lignin content. The expression of PUMYB169 was similar to that of lignin biosynthesisrelated genes, supporting the viewpoint that MYB169 is a transcriptional activator of lignin biosynthesis in fruit stone cells.

To further examine the relationship between ROS accumulation and stone cell formation, we examined $\mathrm{H}_{2}$ DCF-DA-stained fruit sections at the cellular level via confocal microscopy. Confocal microscopy indicated strong $\mathrm{H}_{2}$ DCF-DA fluorescence at stone cell positions (Fig. 2a), which is consistent with the ROS fluorescence positions. The intensity of the fluorescence signal increased with increasing stone cell formation.
Furthermore, TEM was performed on cerium chloridestained sections to determine the subcellular localization of ROS species during stone cell formation. There was high accumulation of $\mathrm{H}_{2} \mathrm{O}_{2}$ in the samples, as indicated by the obvious accumulation of black spots in the secondary cell walls of stone cells (Fig. 2b). These observations further supported the correlation between stone cell lignification and ROS accumulation.

\section{Involvement of ROS signaling in stone cell formation}

$\mathrm{NADPH}$ oxidase activation during defensive responses is a key enzymatic source of ROS in plants. To evaluate the role of NADPH oxidase as an ROS source during lignin biosynthesis, pear fruit were injected with $\mathrm{H}_{2} \mathrm{O}_{2}$ and DPI (an NADPH oxidase inhibitor) at 40 DAFB. Ten days after injection, relative to that in the control treatment, lignin staining was significantly lower at the infiltration sites in the DPI treatment and significantly higher at the infiltration sites in the $\mathrm{H}_{2} \mathrm{O}_{2}$ treatment (Fig. 3a). Moreover, both DAB and $\mathrm{H}_{2}$ DCF-DA staining were performed to analyze ROS production. DAB staining revealed small quantities of ROS in the DPI treatment group but significant ROS accumulation in the $\mathrm{H}_{2} \mathrm{O}_{2}$ treatment group (Fig. 3b). Similarly, weaker $\mathrm{H}_{2}$ DCF-DA fluorescence was observed at the stone cell positions in the DPItreated samples than in the control samples (Fig. 3d).

Compared with that of the samples in the control group, the lignin content of the $\mathrm{H}_{2} \mathrm{O}_{2}$-treated samples was $22.13 \%$ higher, while that of DPI-treated samples was $23.39 \%$ lower. Similarly, the NADPH oxidase activity of the DPI-treated samples was significantly lower than that of the samples in the control group. Moreover, the accumulation of $\mathrm{H}_{2} \mathrm{O}_{2}$ in the flesh tissue of DPI-treated samples was lower than that of samples in the control group (Fig. 3d). Thus, applying an NADPH oxidase inhibitor inhibited lignin formation and $\mathrm{H}_{2} \mathrm{O}_{2}$ generation in the pear fruit. These data suggest that NADPH oxidase produces ROS, which plays a pivotal role in the lignification of stone cells.

\section{Identification of $\mathrm{RBOH}$ genes and transcripts during stone cell formation}

Based on similarity searches using Arabidopsis and Malus $R B O H$ gene sequences ${ }^{45,46}$, eight homologous $\mathrm{RBOH}$ genes were identified from pear genomes. Phylogenetic analysis was performed to elucidate the evolutionary relationship of pear, apple, and Arabidopsis genes (Fig. 4a). The PuRBOH genes were classified into four subgroups of genes whose sequences were closely linked to the homologous sequences of $A t R B O H D,-E,-F$ and $-H$.

To identify the $R B O H$ genes involved in stone cell formation, we examined $R B O H$ gene expression in the fruit at six developmental stages using qRT-PCR (Fig. 4b). Among the eight pear $R B O H$ genes, $P u R B O H D 3$ and 


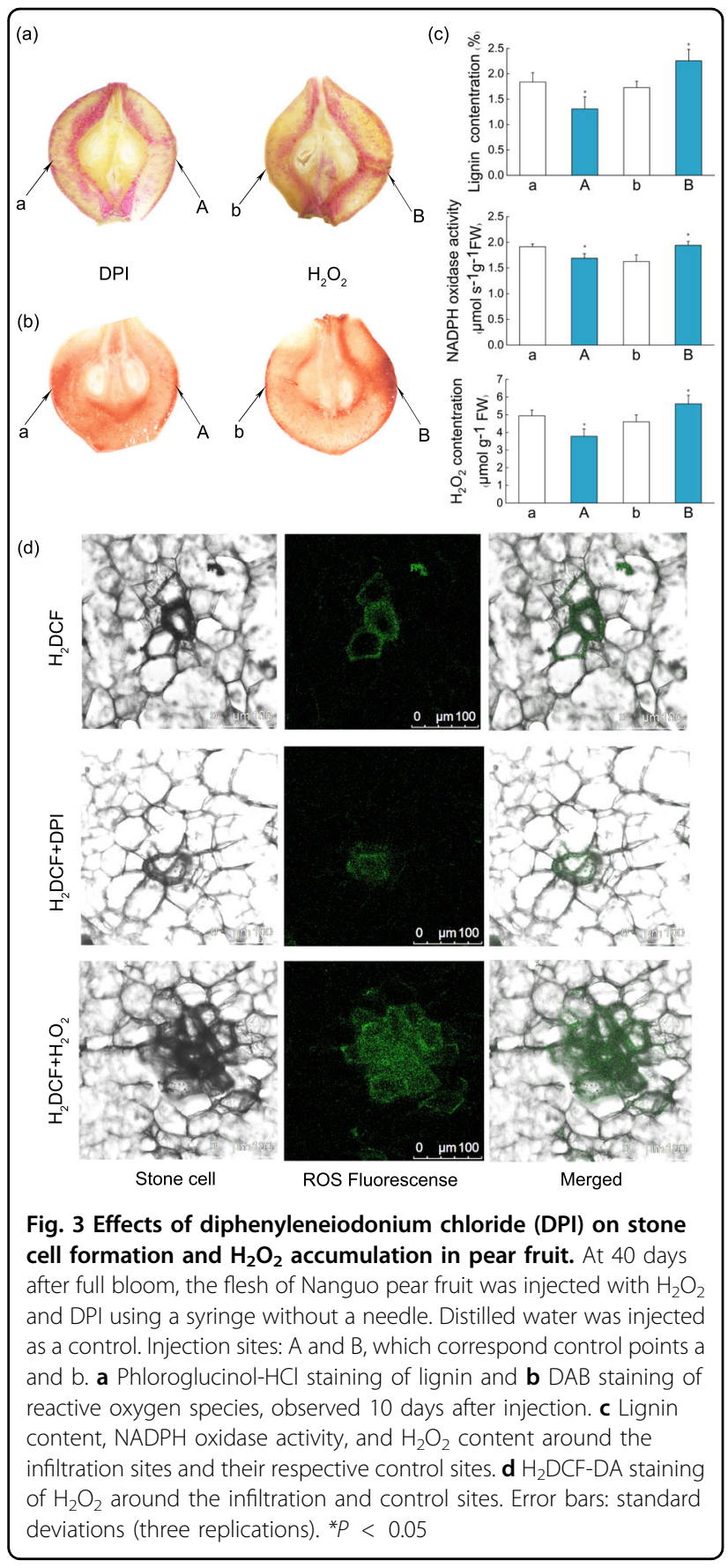

PuRBOHF were upregulated at the early stages of pear fruit development but expressed at low levels at the later stage, which was similar to the changes in lignin and ROS levels. To further confirm the relationships between the expression of $P u R B O H D 3$ and PuRBOHF and stone cell formation, stone cells were isolated from flesh tissues at 40 DAFB and subjected to qRT-PCR analysis. The results showed that PuRBOHF was expressed at the highest levels in isolated stone cells (Fig. 4c), indicating that this gene is the most likely $R B O H$ member to participate in the formation of stone cells in pear.

\section{Expression pattern of PuRBOHF}

To further examine the spatial expression patterns of $P U R B O H F$ in pear fruit, we performed RNA in situ hybridization with a $P u R B O H F$-specific probe using flesh tissues at 40 DAFB. We found that PuRBOHF preferentially accumulated in the stone cell zone and found a relatively weak signal in the parenchymal cells, suggesting that this gene is involved in stone cell formation (Fig. 4d). To examine the tissue specificities of $P u R$ $B O H F$, comparative gene expression analysis was performed using different tissues of Nanguo plants. Among these tissues, PuRBOHF was predominantly transcribed in the leaves and young fruits (Fig. 4e). To analyze the response pattern of PuRBOHF to various abiotic stresses and hormone treatments, gene expression analysis was performed using Nanguo pear fruit at 40 DAFB. Compared to their levels in the untreated fruits, $P u R B O H F$ transcript levels in response to abiotic treatments (chilling and wounding) as well as ABA, SA, and ETH treatments significantly increased (Fig. 4f), suggesting that $P U R B O H F$ is involved in the pear stress response. For the subcellular localization analysis of PuRBOHF, the green fluorescence signal of 35S-GFP was distributed throughout the cell, whereas the signals from the PuRBOHF-GFP construct were localized in the plasma membrane, indicating that PuRBOHF localized to the plasma membrane (Fig. S2).

\section{Transient expression of PuRBOHF in pear fruit}

To further elucidate the function of $P u R B O H F$ in lignin biosynthesis, PuRBOHF overexpression or antisense constructs were transferred into Nanguo pear fruit at 40 DAFB. Ten days after infiltration, the lignin staining was darker in the PuRBOHF-overexpressing samples than in the control samples (Fig. 5a). Moreover, there was a decrease in the staining intensity of the flesh at the injection site following the silencing of $P u R B O H F$. There was a $74.51 \%$ increase in the lignin content of the flesh around the infiltration sites compared with that around the noninjected sites (Fig. 5d). However, the silencing of PURBOHF induced a significant decrease in lignin content. DAB-stained tissue sections were also examined to determine the effects of PuRBOHF overexpression or silencing on ROS generation. The amount of brown precipitates in the sites injected with the PuRBOHF overexpression vector was greater than that at the corresponding noninjected sites. In contrast, diluted brown precipitates were observed at the site infiltrated with the PuRBOHF silencing vector (Fig. 5b). The $\mathrm{H}_{2} \mathrm{O}_{2}$ levels were consistent with the histochemical staining results. Taken together, these results showed that the overexpression 


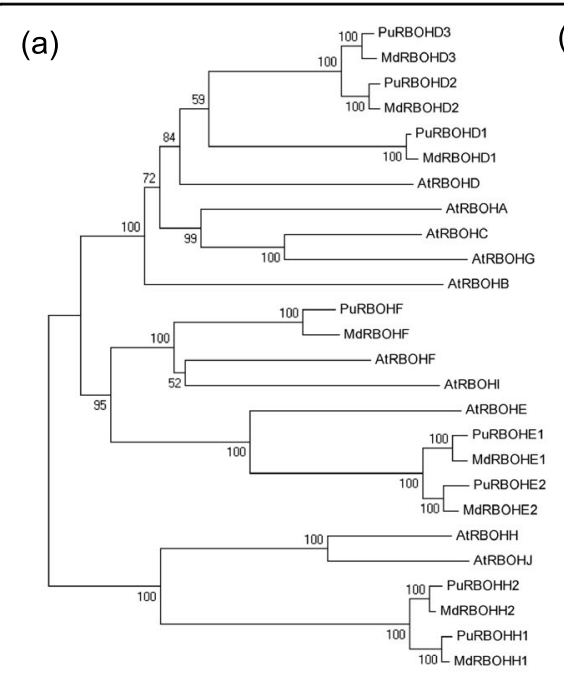

(d)

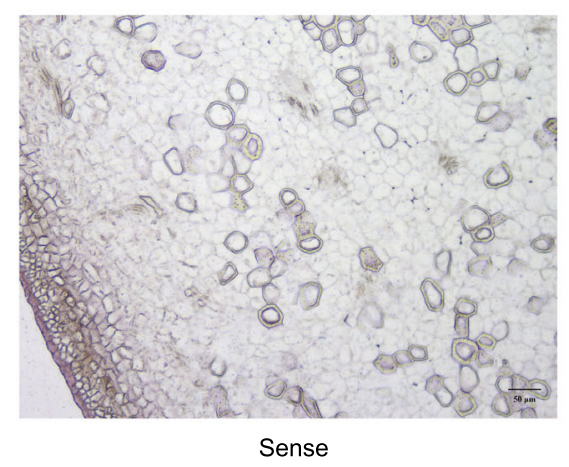

(b)
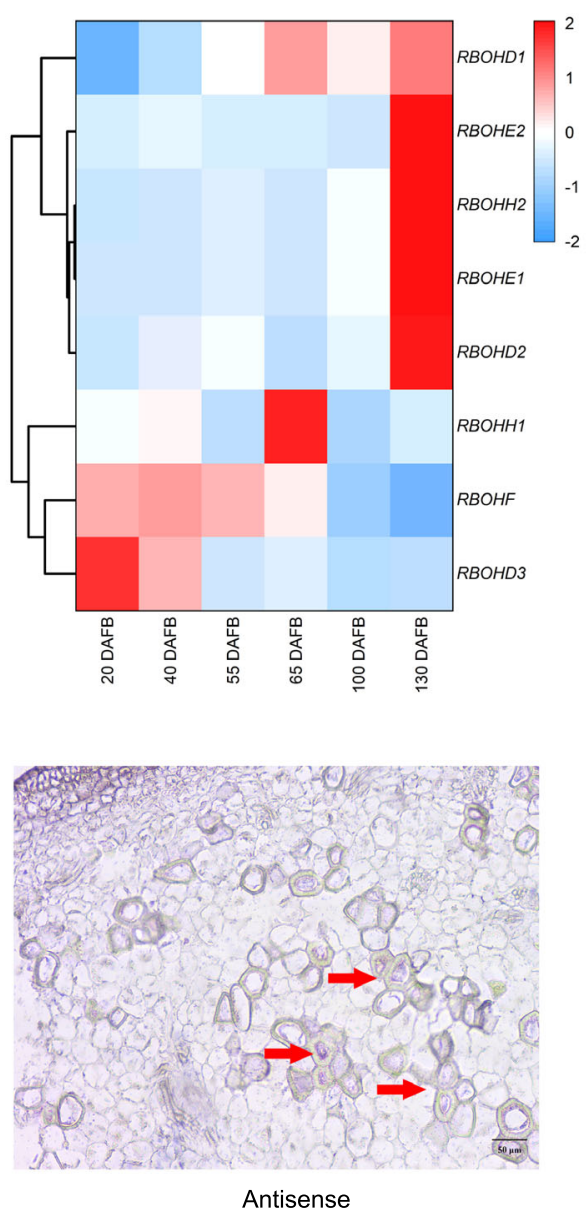

(c)

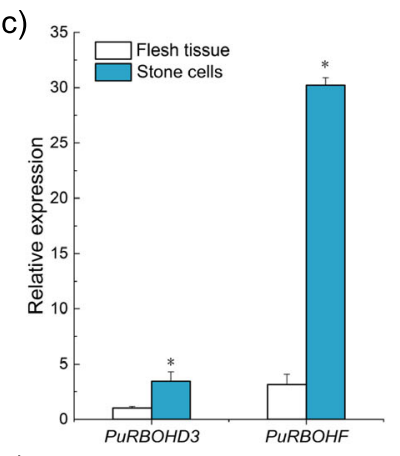

(e)

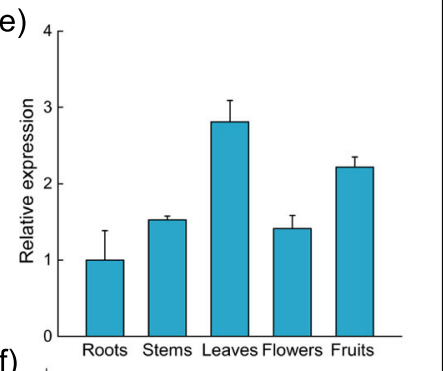

(f)

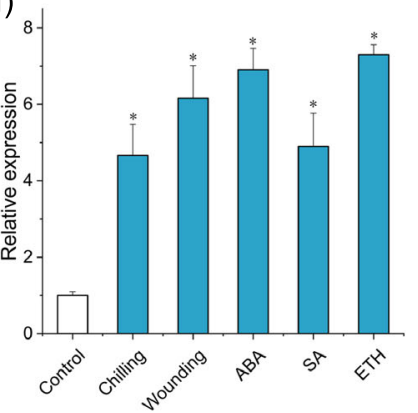

Fig. 4 Phylogenetic relationships among RBOHs and relative PuRBOHF expression levels. a Phylogenetic tree of $R B O H$ sequences of Arabidopsis thaliana (AtRBOH), Malus domestica (MdRBOH), and Pyrus ussuriensis (PuRBOH) based on a conservative approximate alignment. The scale indicates the genetic distance. $\mathbf{b}$ Heatmap of the qRT-PCR-derived cluster analysis of eight PuRBOH genes expressed in fruit flesh. $\mathbf{c}$ Relative transcript levels of PURBOHD3 and PURBOHF in stone cells and fruit flesh tissues at 40 days after full bloom (DAFB). $\mathbf{d}$ RNA in situ hybridization of PuRBOHF in fruit flesh tissues at 40 DAFB, cross-sectioned for hybridization with sense (left column) and antisense (right column) probes. Red triangles: in situ hybridization signals of PURBOHF transcripts. e PURBOHF expression in various tissues. $\mathbf{f}$ PURBOHF expression under various abiotic stresses and hormone treatments. Error bars: standard deviations (three replications). ${ }^{*} P<0.05$

and silencing of PuRBOHF affected lignin accumulation in pear fruit.

\section{Overexpression of PURBOHF in pear calli}

The function of PURBOHF was further confirmed by overexpressing this gene in pear calli induced from Nanguo pear flesh. Excessive lignin accumulation was observed in PuRBOHF-overexpressing calli compared to wild-type calli after 30 days (Fig. $5 \mathrm{f}$ ). The transcript level of $P u R B O H F$ also significantly increased in PuRBOHF-overexpressing calli compared with wild-type calli (Fig. 5e). Furthermore, the expression of lignin synthesis-related genes was analyzed in empty vector-transformed and PuRBOHF-overexpressing calli. Consistent with the increased lignin content, PuRBOHF overexpression also resulted in a significant increase in the transcription of monolignol biosynthesis-related genes, and PuPOD2 and PuLAC2 exhibited the highest increases (Fig. 5g). Thus, these genes may play key roles in PuRBOHF-mediated lignin biosynthesis. Taken together, these results further confirmed that $P U R B O H F$ plays an essential role in lignin biosynthesis in pear fruit.

\section{$\mathrm{H}_{2} \mathrm{O}_{2}$ triggers PuPOD2 and PuLAC2 promoter activity}

POD and LAC have been shown to catalyze the formation of lignin polymers using monolignols and $\mathrm{ROS}^{13}$. In the present study, there was an increase in the expression of PuPOD2 and PuLAC2 in PuRBOHF-overexpressing calli. To examine the effects of $\mathrm{H}_{2} \mathrm{O}_{2}$ on PuPOD2 and PuLAC2 promoter activity, proPuPOD-GUS and proPuLAC2-GUS containing GV1301 cells were infiltrated into tobacco leaves. $\mathrm{H}_{2} \mathrm{O}_{2}$ treatment of the 


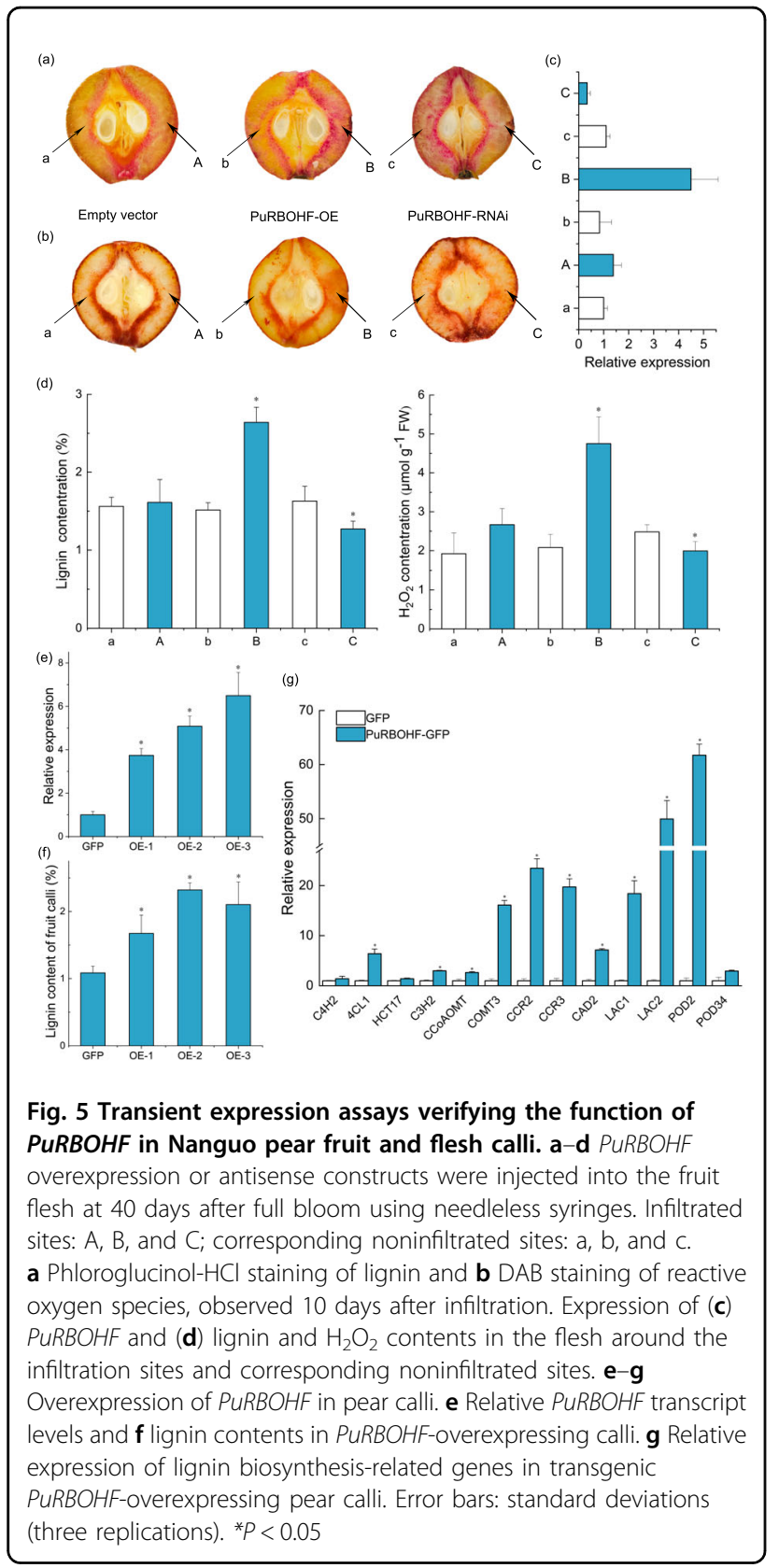

infiltrated leaves caused a significant increase in GUS staining retention compared with that of leaves in the control group (Fig. 6b). Consistent with these results, enhanced GUS activity was observed after treatment with $\mathrm{H}_{2} \mathrm{O}_{2}$, indicating that $\mathrm{H}_{2} \mathrm{O}_{2}$ induced the transcription of PuPOD2 and PuLAC2.

\section{PuMYB169 activates the PuRBOHF promoter}

To explore whether PuMYB169 affects the transcriptional activity of the PuRBOHF promoter, we cloned $1499 \mathrm{bp}$ of the promoter sequence upstream from the ATG codon of $P u R B O H F$, inserted this region upstream
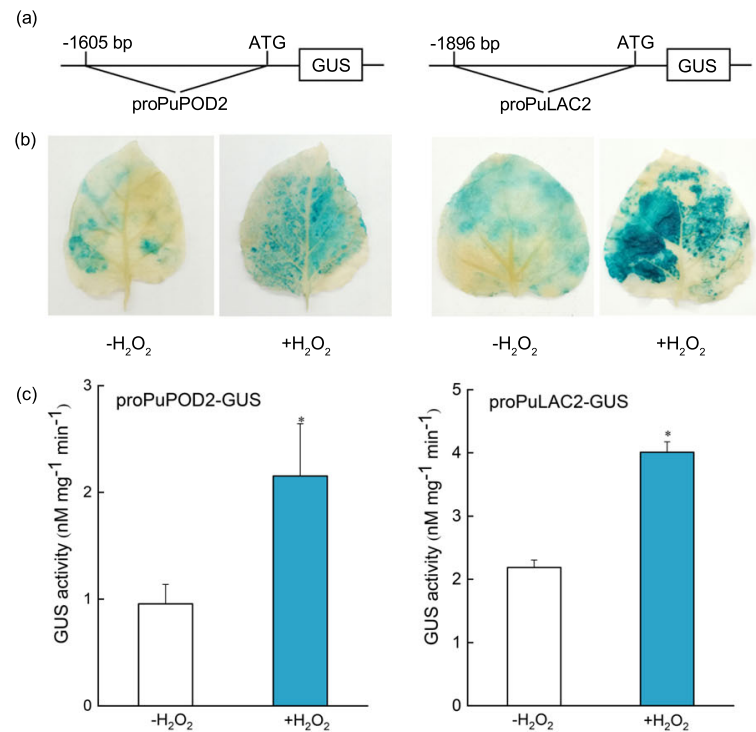

(d) Effector
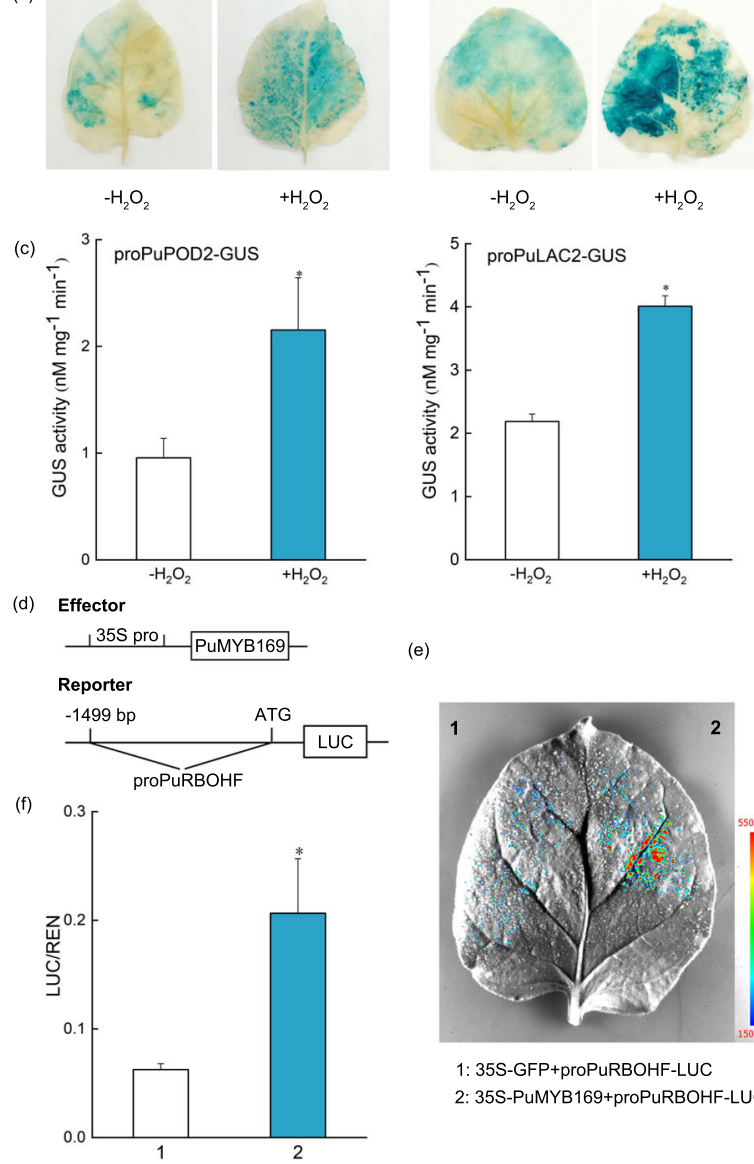

(e)

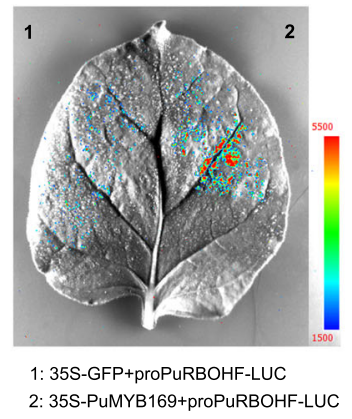

Fig. 6 Relationship between PURBOHF-mediated ROS production and the stone cell transcriptional network. a-c Effects of $\mathrm{H}_{2} \mathrm{O}_{2}$ on proPUPOD2-GUS and proPuLAC2-GUS promoter activity. a Schematics of GUS reporter gene constructs driven by the PUPOD2 promoter or PULAC2 promoter. The constructs were transformed into 5-week-old tobacco leaves. The infiltrated tobacco leaves were then sprayed with $\mathrm{H}_{2} \mathrm{O}_{2}$ solution $\left(+\mathrm{H}_{2} \mathrm{O}_{2}\right)$ or distilled water $\left(-\mathrm{H}_{2} \mathrm{O}_{2}\right)$. After 3 days of growth, the leaves were harvested for $\mathbf{b}$ histochemical GUS staining and $\mathbf{c}$ GUS activity analysis. d-f PUMYB169 activated PURBOHF transcription, as revealed by a dual-luciferase (LUC) assay.

d Schematics of the reporter and effector gene constructs used in the dual LUC assay. e In tobacco leaves, transient expression of PUMYB169 activated PURBOHF promoter activity. The transient expression assay was performed with three replicates. $\mathbf{f}$ Quantitative analysis of the luminescence intensity for each image in (e). Error bars: standard deviations (three replications). ${ }^{*} P<0.05$

of a $L U C$ reporter gene and cotransfected effector plasmids harboring the resulting 35S-PuMYB169 construct into tobacco leaves. The luciferase assays revealed that, compared with expression of the PuRBOHF promoter alone, coexpression of 35S-PuMYB169 and 
proPuRBOHF-LUC resulted in much stronger luminescence (Fig. 6e). These results indicated that PuMYB169 directly activated the expression of PuRBOHF.

\section{Discussion}

NADPH oxidase-mediated ROS production is essential for stone cell formation

Stone cell content and size are key factors determining the internal quality of pear fruit ${ }^{47}$. ROS are important signaling molecules involved in development and stress responses in plants ${ }^{48}$. In this study, we found that the accumulation of ROS was closely associated with stone cell lignification in pear fruit. During the fruit development period, we observed similar trends of stone cell, lignin, and $\mathrm{H}_{2} \mathrm{O}_{2}$ contents. Moreover, confocal microscopy and TEM imaging results further demonstrated the spatial consistency between $\mathrm{H}_{2} \mathrm{O}_{2}$ and lignification of the cell wall. These results indicate that ROS accumulation is closely related to stone cell development and are consistent with the findings of Heng et al. ${ }^{23}$. The exocarp cell $\mathrm{H}_{2} \mathrm{O}_{2}$ concentration was strongly positively associated with the lignin concentration.

Stone cell lignification in pear fruit was reduced by DPI. Moreover, NADPH oxidase activity and $\mathrm{H}_{2} \mathrm{O}_{2}$ levels were suppressed by DPI. These results further support the hypothesis that NADPH oxidase-mediated ROS production is essential for lignin biosynthesis in pear fruit. Pretreatment with DPI was shown to inhibit lignin deposition induced by cell wall damage in root tips ${ }^{49}$ and suppressed lignin accumulation in senescing cells in the abscission zone in Arabidopsis ${ }^{50}$. Moreover, the application of $\mathrm{H}_{2} \mathrm{O}_{2}$ promotes lignin formation in rice roots ${ }^{22}$. Taken together, these findings indicate that $\mathrm{RBOH}$-mediated ROS production is responsible for stone cell lignification.

\section{Functional conservation and diversity of PuRBOHF genes}

NADPH oxidases are encoded by $R B O H$ genes, which are present in a wide range of plant species ${ }^{51}$. However, the role of $\mathrm{RBOHs}$ in lignification in vivo is still under investigation. AtRBOHD and $A t R B O H F$ are closely related to lignification in Arabidopsis. There was a severe delay in the formation of a function Casparian strip in $R B O H F$ knockdown mutants, indicating that $R B O H F$ plays a role in the formation of lignin-containing Casparian strips that function as diffusion barriers in root endodermal cells ${ }^{21}$. Furthermore, Arabidopsis RBOHD/F knockdown mutants were shown to be unable to accumulate lignin in the floral

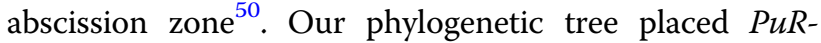
$B O H F$ in the same clade as AtRBOHF. The role of these $R B O H \mathrm{~s}$ in ROS-mediated lignin accumulation in stone cells remains unclear.

It has recently been shown that PbRBOHA and $P b R B O H D$ expression is consistent with the increased ROS content during stone cell formation in pear fruit ${ }^{28}$.
However, there is no clear spatiotemporal evidence of $\mathrm{RBOH}$ involvement in the lignification of stone cells. In the present study, in situ hybridization analysis showed an increase in the expression of $P U R B O H F$ in developing stone cells, indicating the involvement of $P u R B O H F$ in the lignification of pear fruit stone cells. Moreover, $P u R B O H F$ was localized to the plasma membrane, including sites at which lignification occurred. Therefore, we speculated that $P U R B O H F$ is a putative candidate gene involved in the lignin synthesis pathway in pear fruit.

To further characterize PuRBOHF functions during stone cell formation, a transient transformation assay was performed on pear fruit and calli. PuRBOHF-overexpressing pear fruit and calli produced higher ROS and lignin contents than did fruits in the control groups. In addition, qRT-PCR analysis indicated that the expression levels of lignin biosynthesis-related genes, including PuPOD2 and PuLAC2, were significantly upregulated in PuRBOHF-overexpressing calli. However, suppression of PuRBOHF expression by VIGS repressed lignin and ROS accumulation. Taken together, these results suggest that PuRBOHF plays an essential role in ROS formation during pear stone cell formation.

\section{PuRBOHF and the stone cell transcriptional network}

ROS play a catalytic role in lignification via oxidative polymerization of monolignols to $\operatorname{lignin}{ }^{13} \cdot \mathrm{H}_{2} \mathrm{O}_{2}$ is a major ROS involved in the lignification process. In addition to its key role as a cosubstrate, $\mathrm{H}_{2} \mathrm{O}_{2}$ may also play a regulatory role in cell signaling ${ }^{52}$. The activities of PAL, $\mathrm{C} 4 \mathrm{H}$, and $4 \mathrm{CL}$ are reportedly induced by endogenous $\mathrm{H}_{2} \mathrm{O}_{2}{ }^{10}$. However, the relationship between $\mathrm{H}_{2} \mathrm{O}_{2}$ accumulation and stone cell lignification has not been previously clarified. In the present study, the expression of PuPOD2 and PuLAC2 was upregulated in PuRBOHFoverexpressing calli. Furthermore, the results of our GUS assay showed that the response of PuPOD2 and PuLAC2 to $\mathrm{H}_{2} \mathrm{O}_{2}$ was regulated at the promoter level. These results revealed that $\mathrm{RBOH}$-derived $\mathrm{H}_{2} \mathrm{O}_{2}$ not only played a catalytic role in lignification but also induced the expression of genes regulating the polymerization of monolignols to lignin.

Xue et al. ${ }^{4}$ identified MYB169 as a positive regulatory transcription factor involved in lignin biosynthesis in fruit stone cells. In addition, MYB169 activates the transcription of $C 3 H, C C R$, and $C A D$. In the present study, PuMYB169 directly activated the transcription of $P u R$ $B O H F$. Our findings thus showed that, by inducing $P u R$ $B O H F$ expression, PuMYB169 is involved in the regulation of the lignin polymerization process. However, the mechanism underlying the interaction between PuMYB169 and PuRBOHF requires further verification.

The presence of lignified stone cells substantially reduce fruit quality. Therefore, it is desirable to inhibit stone cell 


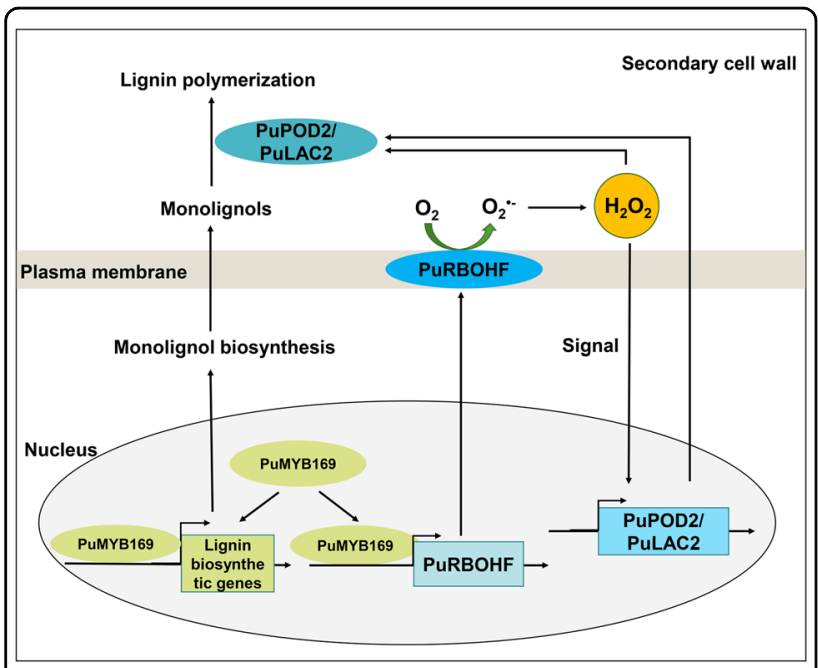

Fig. 7 Model of the PURBOHF-mediated reactive oxygen species (ROS) signaling pathway involved in stone cell lignification in pear fruit. PURBOHF, controlled by PUMYB169, enhances the ROS burst in the plasma membrane. ROS then serve as secondary messengers and induce the transcription of PUPOD2 and PULAC2, ultimately promoting monolignol polymerization and stone cell formation. In addition, apoplastic ROS are necessary for the oxidative polymerization of monolignols to lignin

development to produce high-quality pear fruit. Previous studies have shown that ROS are involved in the lignin polymerization process ${ }^{13}$. The mechanism underlying the activities of ROS in lignification was elucidated in the present study. We found that, in pear fruit, stage-specific PuRBOHF expression contributed to a temporal peak in ROS production, which is closely associated with stone cell lignification. In turn, stone cell lignification is regulated by the upregulation of PuRBOHF expression, which is controlled by PuMYB169 (Fig. 7). PuRBOHF, in turn, induces apoplastic $\mathrm{O}_{2}$ production, which is subsequently spontaneously or enzymatically converted to $\mathrm{H}_{2} \mathrm{O}_{2}$. The $\mathrm{H}_{2} \mathrm{O}_{2}$ is then transported into the cytosol, where it induces PuPOD2 and PULAC2 transcription. Apoplastic ROS are also necessary for the oxidative polymerization of monolignols into lignin. Overall, the findings of the present study improve the knowledge of the underlying mechanism and signaling pathways involved in the regulation of stone cell lignification in pear fruit.

\section{Acknowledgements}

We thank A.P. Biao Wang for technical assistance and discussion of the results. This work was supported by the National Natural Science Foundation of China (31701865), the Liaoning Provincial Natural Science Foundation of China (2019MS-276), the Research Foundation of the Education Bureau of Liaoning Province and the China Agriculture Research System of MOF and MARAE.

\section{Author contributions}

X.W. designed the study and wrote the manuscript; G.D. and X.W. obtained the funding; S.L., H.S., and Y.L. carried out the experiments; C.L. analyzed the data; and G.D., D.L., and X.L. provided suggestions. All the authors have read and approved the final manuscript.

\section{Data availability}

Supporting data are included within the article and its supplementary information. Other relevant materials are available from the corresponding author upon request.

\section{Conflict of interest}

The authors declare no competing interests.

Supplementary information The online version contains supplementary material available at https://doi.org/10.1038/s41438-021-00674-0.

Received: 10 May 2021 Revised: 13 July 2021 Accepted: 30 July 2021

Published online: 01 December 2021

\section{References}

1. Bassil, N. \& Postman, J. D. Identification of European and Asian pears using EST-SSRs from Pyrus. Genet. Resour. Crop Ev 57, 357-370 (2010).

2. Liu, Q. et al. Genetic diversity and population structure of pear (Pyrus spp.) collections revealed by a set of core genome-wide SSR markers. Tree Genet. Genomes 11, 1-22 (2015).

3. Yan, C. et al. Stone cell distribution and lignin structure in various pear varieties. Sci. Hortic.- Amst. 174, 142-150 (2014).

4. Xue, C. et al. PbrMYB169 positively regulates lignification of stone cells in pear fruit. J. Exp. Bot. 70, 1801-1814 (2019).

5. Cai, Y. et al. Study of the structure and biosynthetic pathway of lignin in stone cells of pear. Sci. Hortic. 125, 374-379 (2010).

6. Li, S. H., Schneider, B. \& Gershenzon, J. Microchemical analysis of lasermicrodissected stone cells of Norway spruce by cryogenic nuclear magnetic resonance spectroscopy. Planta 225, 771-779 (2007).

7. Choi, J. \& Lee, S. Distribution of stone cell in Asian, Chinese, and European pear fruit and its morphological changes. J. Appl Bot. Food Qual. 86, 185-189 (2013).

8. Shinya, T. et al. Transcriptional profiles of hybrid Eucalyptus genotypes with contrasting lignin content reveal that monolignol biosynthesis-related genes regulate wood composition. Front. Plant Sci. 7, 443 (2016).

9. Gong, $X$. et al. PbMCla/1b regulates lignification during stone cell development in pear (Pyrus bretschneideri) fruit. Hortic. Res.-Engl. 7, 1-13 (2020).

10. Li, D. et al. Hydrogen peroxide accelerated the lignification process of bamboo shoots by activating the phenylpropanoid pathway and programmed cell death in postharvest storage. Postharvest Biol. Tec. 153, 79-86 (2019).

11. Yoon, J., Choi, H. \& An, G. Roles of lignin biosynthesis and regulatory genes in plant development. J. Integr. Plant Biol. 57, 902-912 (2015).

12. Wang, Y., Chantreau, M., Sibout, R. \& Hawkins, S. Plant cell wall lignification and monolignol metabolism. Front Plant Sci. 4, 220 (2013).

13. Barros, J., Serk, H., Granlund, I. \& Pesquet, E. The cell biology of lignification in higher plants. Ann. Bot. 115, 1053-1074 (2015).

14. Chen, Q. \& Yang, G. Signal function studies of ROS, especially RBOHdependent ROS, in plant growth, development and environmental stress. J. Plant Growth Regul. 39, 157-171 (2020).

15. Orman-Ligeza, B. et al. RBOH-mediated ROS production facilitates lateral root emergence in Arabidopsis. Development 143, 3328-3339 (2016).

16. Sierla, M., Waszczak, C., Vahisalu, T. \& Kangasjärvi, J. Reactive oxygen species in the regulation of stomatal movements. Plant Physiol. 171, 1569-1580 (2016).

17. Singh, R. et al. Reactive oxygen species (ROS): beneficial companions of plants' developmental processes. Front Plant Sci. 7, 1299 (2016).

18. Xie, H. T., Wan, Z. Y., Li, S. \& Zhang, Y. Spatiotemporal production of reactive oxygen species by NADPH oxidase is critical for tapetal programmed cell death and pollen development in Arabidopsis. Plant Cell 26, 2007-2023 (2014).

19. Yang, $X$. et al. The NADPH-oxidase LsRbohC1 plays a role in lettuce (Lactuca sativa) seed germination. Plant Physiol. Bioch. 154, 751-757 (2020).

20. Yamauchi, T. et al. An NADPH oxidase $\mathrm{RBOH}$ functions in rice roots during lysigenous aerenchyma formation under oxygen-deficient conditions. Plant Cell 29, 775-790 (2017).

21. Lee, Y., Rubio, M. C., Alassimone, J. \& Geldner, N. A mechanism for localized lignin deposition in the endodermis. Cell 153, 402-412 (2013).

22. Liu, Q. et al. Transcriptional and physiological analyses identify a regulatory role for hydrogen peroxide in the lignin biosynthesis of copper-stressed rice roots. Plant Soil 387, 323-336 (2015). 
23. Heng, W. et al. Relationship between $\mathrm{H}_{2} \mathrm{O}_{2}$ in polyamine metabolism and lignin in the exocarp of a russet mutant of 'Dangshansuli' pear (Pyrus bretschneideri Rehd.). Plant Mol. Biol. Rep. 34, 1056-1063 (2016).

24. Liu, Y. \& He, C. Regulation of plant reactive oxygen species (ROS) in stress responses: learning from AtRBOHD. Plant Cell Rep. 35, 995-1007 (2016).

25. Lee, D. et al. Regulation of reactive oxygen species during plant immunity through phosphorylation and ubiquitination of RBOHD. Nat. Commun. 11 1-16 (2020).

26. Müller, K., Carstens, A. C., Linkies, A., Torres, M. A. \& Leubner-Metzger, G. The $\mathrm{NADPH}$-oxidase AtrbohB plays a role in Arabidopsis seed after-ripening. $N$. Phytol. 184, 885-897 (2009).

27. Foreman, J. et al. Reactive oxygen species produced by NADPH oxidase regulate plant cell growth. Nature 422, 442-446 (2003).

28. Cheng, $X$. et al. In silico genome-wide analysis of respiratory burst oxidase homolog $(\mathrm{RBOH})$ family genes in five fruit-producing trees, and potential functional analysis on lignification of stone cells in chinese white pear. Cells $\mathbf{8}$, 520 (2019).

29. Zhang, J. et al. Comparison of the transcriptomic analysis between two Chinese white pear (Pyrus bretschneideri Rehd.) genotypes of different stone cells contents. PLOS ONE 12, e0187114 (2017).

30. Anderson, N. A. et al. Manipulation of guaiacyl and syringyl monomer biosynthesis in an Arabidopsis cinnamyl alcohol dehydrogenase mutant results in atypical lignin biosynthesis and modified cell wall structure. Plant Cell 27 2195-2209 (2015).

31. Tao, S., Khanizadeh, S., Zhang, H. \& Zhang, S. Anatomy, ultrastructure and lignin distribution of stone cells in two Pyrus species. Plant Sci. 176, 413-419 (2009).

32. Brennan, T. \& Frenkel, C. Involvement of hydrogen peroxide in the regulation of senescence in pear. Plant Physiol. 59, 411-416 (1977).

33. Choi, H. W., Kim, Y. J., Lee, S. C., Hong, J. K. \& Hwang, B. K. Hydrogen peroxide generation by the pepper extracellular peroxidase $\mathrm{CaPO}_{2}$ activates local and systemic cell death and defense response to bacterial pathogens. Plant Physiol. 145, 890-904 (2007)

34. Chen, Z. et al. The AtrbohF-dependent regulation of ROS signaling is required for melatonin-induced salinity tolerance in Arabidopsis. Free Radic. Bio Med. 108, 465-477 (2017)

35. Bestwick, C. S., Brown, I. R., Bennett, M. H. \& Mansfield, J. W. Localization of hydrogen peroxide accumulation during the hypersensitive reaction of lettuce cells to Pseudomonas syringae pv phaseolicola. Plant Cell 9, 209-221 (1997).

36. Zhang, J. et al. Reactive oxygen species produced via plasma membrane NADPH oxidase regulate anthocyanin synthesis in apple peel. Planta $\mathbf{2 4 0}$ 1023-1035 (2014).
37. Sagi, M. \& Fluhr, R. Superoxide production by plant homologues of the gp91phox NADPH oxidase. Modulation of activity by calcium and by tobacco mosaic virus infection. Plant Physiol. 126, 1281-1290 (2001).

38. Cheng, $X$. et al. Characterization and analysis of CCR and CAD gene families at the whole-genome level for lignin synthesis of stone cells in pear (Pyrus bretschneideri) fruit. Biol. open. 6, 1602-1613 (2017)

39. Xue, C. et al. PbrmiR397a regulates lignification during stone cell development in pear fruit. Plant Biotechnol. J. 17, 103-117 (2019a).

40. Bai, S. et al. BBX16, a B-box protein, positively regulates light-induced anthocyanin accumulation by activating MYB10 in red pear. Plant Biotechnol. J. 17 1985-1997 (2019).

41. Wu, A. et al. JUNGBRUNNEN1, a reactive oxygen species-responsive NAC transcription factor, regulates longevity in Arabidopsis. Plant Cell. 24, 482-506 (2012).

42. He, J. et al. DEXH Box RNA helicase-mediated mitochondrial reactive oxygen species production in Arabidopsis mediates crosstalk between abscisic acid and auxin signaling. Plant Cell. 24, 1815-1833 (2012).

43. Jefferson, R. A., Kavanagh, T. A. \& Bevan, M. W. GUS fusions: beta-glucuronidase as a sensitive and versatile gene fusion marker in higher plants. EMBO J. $\mathbf{6}$, 3901-3907 (1987)

44. Yang, C. et al. Activation of ethylene signaling pathways enhances disease resistance by regulating ROS and phytoalexin production in rice. Plant J. $\mathbf{8 9}$ 338-353 (2017)

45. Torres, M. A. \& Dangl, J. L. Functions of the respiratory burst oxidase in biotic interactions, abiotic stress and development. Curr. Opin. Plant Biol. 8, 397-403 (2005).

46. Cepauskas, D. et al. Characterization of apple NADPH oxidase genes and their expression associated with oxidative stress in shoot culture in vitro. Plant Cell, Tiss. Org. 124, 621-633 (2016).

47. Wang, X. et al. Biochemical characterization and expression analysis of lignification in two pear (Pyrus ussuriensis Maxim.) varieties with contrasting stone cell content. Protoplasma 257, 261-274 (2020).

48. Gayomba, S. R. \& Muday, G. K. Flavonols regulate root hair development by modulating accumulation of reactive oxygen species in the root epidermis. Development 147, dev185819 (2020).

49. Denness, L. et al. Cell wall damage-induced lignin biosynthesis is regulated by a reactive oxygen species-and jasmonic acid-dependent process in Arabidopsis. Plant Physiol. 156, 1364-1374 (2011).

50. Lee, Y. et al. A lignin molecular brace controls precision processing of cell walls critical for surface integrity in Arabidopsis. Cell 173, 1468-1480 (2018).

51. Kärkönen, A. \& Kuchitsu, K. Reactive oxygen species in cell wall metabolism and development in plants. Phytochemistry 112, 22-32 (2015).

52. Barceló, A. R. Xylem parenchyma cells deliver the $\mathrm{H}_{2} \mathrm{O}_{2}$ necessary for lignification in differentiating xylem vessels. Planta 220, 747-756 (2005). 